\title{
Bottom-type scattering layers and equatorial spread $F$
}

\author{
D. L. Hysell ${ }^{1}$, J. Chun ${ }^{1}$, and J. L. Chau ${ }^{2}$ \\ ${ }^{1}$ Department of Earth and Atmospheric Sciences, Cornell University, Ithaca, New York, USA \\ ${ }^{2}$ Radio Observatorio de Jicamarca, Instituto Geofísico del Perú, Lima, Peru
}

Received: 7 April 2004 - Revised: 20 August 2004 - Accepted: 25 August 2004 - Published: 22 December 2004

\begin{abstract}
Jicamarca radar observations of bottom-type coherent scattering layers in the post-sunset bottomside F-region ionosphere are presented and analyzed. The morphology of the primary waves seen in radar images of the layers supports the hypothesis of Kudeki and Bhattacharyya (1999) that wind-driven gradient drift instabilities are operating. In one layer event when topside spread $F$ did not occur, irregularities were distributed uniformly in space throughout the layers. In another event when topside spread $F$ did eventually occur, the irregularities within the pre-existing bottom-type layers were horizontally clustered, with clusters separated by about $30 \mathrm{~km}$. The same horizontal periodicity was evident in the radar plumes and large-scale irregularities that emerged later in the event. We surmise that horizontal periodicity in bottom-type layer irregularity distribution is indicative of large-scale horizontal waves in the bottomside F-region that may serve as seed waves for large-scale Rayleigh Taylor instabilities.
\end{abstract}

Key words. Ionosphere (equatorial ionosphere; ionospheric irregularties; plasma waves and instabilities)

\section{Introduction}

Bottom-type layers are thin scattering layers that occur regularly after sunset during equinox and December solstice in the bottomside F-region over Jicamarca and that serve as precursors of large-scale equatorial spread F (ESF) disturbances. They were first described by Woodman and La Hoz (1976) working at Jicamarca but are now known to occur in other longitude sectors. While they are not usually associated with strong radio scintillations or other kinds of interference to communications links, they nevertheless signify the presence of plasma instabilities and are indicative of the stability of the post-sunset ionosphere. We study them for insights into how more disruptive, large-scale spread $F$ plasma irregularities initiate.

Correspondence to: D. L. Hysell

(daveh@geology.cornell.edu)
Early insights into the layers came from Zargham and Seyler (1989), who showed, with a nonlocal analysis, that the linear growth rate of the ionospheric interchange instability operating in the bottomside equatorial F-region has a broad maximum centered on wavelengths of about $1 \mathrm{~km}$. In numerical simulations of the instability initialized by broadband noise, such intermediate-scale waves emerged first and continued to dominate large-scale waves unless the latter were initialized preferentially with the introduction of large-scale "seed" irregularities (Zargham, 1988). Based on this finding, Hysell and Seyler (1998) hypothesized that the intermediate-scale waves suppress large-scale wave growth through anomalous diffusion. Using a renormalization group theory and incorporating satellite data taken to be representative of bottomside irregularities, they estimated that the anomalous diffusion coefficient associated with the kilometric irregularities could be five orders of magnitude greater than the classical ambipolar diffusion coefficient and large enough to impede large-scale wave growth. They suggested that bottom-type layers were composed of kilometric primary waves which are too small to be resolved by coherent scatter radars as anything but a continuous, narrow scattering layer in the bottomside F-region.

Another influence on the scale size distribution of the irregularities in the layers is the distribution of conductivity along magnetic flux tubes. Tsunoda (1981) and Kudeki et al. (1982) showed that the post-sunset bottomside F-region flow is dominated by strong shear, with plasma drifting westward and eastward below and near or above the $F$ peak, respectively. Since the thermospheric wind at F-region altitudes is eastward after sunset, the westward bottomside drifts constitute retrograde motion. Haerendel et al. (1992) identified three mechanisms that could cause retrograde motion: vertical winds on flux tubes with significant integrated Hall conductivity, vertical F-region currents forced by the closure of the equatorial electrojet current near the evening terminator, and the E-region dynamo driven by westward lower thermospheric winds. Since the retrograde drifts can persist long after the passage of the evening terminator, Hysell and Burcham (1998) argued that the E-region dynamo must play 
an important role in equatorial electrodynamics. Examining the extensive JULIA radar database at Jicamarca, they also found that the bottom-type layers always inhabit strata drifting westward or, at most, slowly eastward. The layers therefore appear to reside on flux tubes with Pedersen conductivity concentrated in the E- and valley regions.

To explain how the kilometric plasma waves and instabilities in the bottom-type F-region layers are able to avoid being "shorted out" by the E-region conductivity that dominates the DC electrodynamics of the flux tube, Hysell (2000) invoked a nonlocal theory, attributing the electrical decoupling of the waves from the E-region loading to finite parallel wave number effects. A generalized derivation appears in the Appendix and shows how plasma waves with transverse wavelengths of a few kilometers or less can have growth rates comparable to their local growth rates, even if their amplitudes are constrained to vanish at the feet of the magnetic field lines. The analysis supports a picture of bottom-type scattering layers composed of kilometric primary waves on westward-drifting strata unfavorable to large-scale wave growth.

Several investigators have argued that the shear flow in the bottomside should suppress intermediate-scale waves in favor of large-scale wave growth (Perkins and Doles III, 1975; Guzdar et al., 1983; Satyanarayana et al., 1984). However, building on the insights of Fu et al. (1986), Flaherty et al. (1999) pointed out that the shear makes the system nonnormal, and that even if the intermediate-scale eigenmodes are individually damped by shear, a strong transient response can arise at kilometric scale sizes. The lifetime of the transient growth of intermediate-scale waves in the bottomside F-region was found to be long enough to account for bottomtype layers. Shear therefore poses no fundamental problems for the picture of the layers outlined so far.

However, the picture has been updated recently by Kudeki and Bhattacharyya (1999), who used combined coherent and incoherent radar techniques to observe the so-called postsunset vortex flow over Jicamarca. The evening vortex is a flow feature that arises from shear combined with the evening reversal of the zonal electric field that occurs around twilight. It was analyzed theoretically by Haerendel et al. (1992) and is now routinely observed at Jicamarca using the experimental techniques introduced there by Kudeki and Bhattacharyya (1999), who also pointed out that the retrograde motion of the bottomside F-region makes it unstable to horizontal winddriven gradient drift instabilities in the presence of horizontal density gradients, caused in part by the convection associated with the vortex flow. Given typical plasma-neutral differential flow speeds in excess of $100 \mathrm{~m} / \mathrm{s}$, gradient drift instabilities can have growth rates much larger than the maximum anticipated generalized Rayleigh Taylor growth rate. The vortex is well developed when the prereversal enhancement is strong, and by linking the bottom-type layers to the vortex, the picture outlined by Kudeki and Bhattacharyya (1999) also explains why bottom-type layers and more developed spread $F$ share the same climatology.

In this paper, we examine new observations of bottom-type scattering layers made at the Jicamarca Radio Observatory for evidence of primary gradient drift waves. Jicamarca observes coherent backscatter from small-scale field-aligned irregularities, presumed to be generated by intermediate-scale, primary plasma waves via three-wave mode coupling. The small-scale irregularities then serve as tracers of intermediate and large-scale waves. The new observations were made using an aperture synthesis imaging mode capable of resolving kilometric features in the radar illuminated volume in two spatial dimensions. By monitoring the temporal evolution of the radar images, we look for evidence of shear and vortex flow, track the emergence and growth of primary plasma waves and instabilities, and search for a large-scale bottomside structure that could serve as a seed for ESF plumes.

\section{Radar observations}

Figure 1 shows a bottom-type scattering layer observed by the Jicamarca Radio Observatory on 25 April 2000. The observations were made using a high-power ( $\sim 1$ MW peak) transmitter and with $450 \mathrm{~m}(3 \mu \mathrm{s})$ uncoded pulses, with a pulse repetition frequency (PRF) of $100 \mathrm{~Hz}$. The north and south quarters of the main Jicamarca antenna array were used for transmission, and reception was performed using six modules (64ths) of the array with separate receivers and data acquisition channels attached to each. Data from one receiver are shown in Fig. 1. Here, a bottom-type layer appeared shortly after sunset, ascended briefly, descended, and then dissipated. The thickness and lifetime of this layer are typical. In this case, fully-developed spread $F$ did not occur.

A qualitatively different event is shown in Fig. 2 which represents observations from 27 November 2003. The data were collected using 28-bit binary phase coded pulses with a $300 \mathrm{~m}(2 \mu \mathrm{s})$ baud length but with only $\sim 100 \mathrm{~kW}$ peak power. The PRF was again $100 \mathrm{~Hz}$. Transmission and reception were again performed with the quarters and 64ths of the main antenna array, albeit with a slightly different configuration described below. In this event, the bottom-type layer ascended slowly and continuously and was eventually accompanied by some plume structures in the early stage of formation. Topside irregularities passed over the radar after the bottom-type layer dissipated. The former were presumably generated during the hour preceding the time they were observed.

Horizontal streaks in the RTI diagrams hint at the existence of fine structure in the bottom-type layers. The streaks are somewhat more pronounced and widely spaced in the 25 April 2000 data than in the 27 November 2003 data. The significance of the streaks becomes clear with the application of aperture synthesis imaging techniques.

Interferometry with multiple baselines was introduced at Jicamarca to construct true images of the radar targets illuminated by the transmitting antenna beam (Kudeki and Sürücü, 1991). It is well known that interferometry using a single antenna baseline yields two moments of the radio brightness distribution, the distribution of received power versus bearing (Farley et al., 1981). Interferometry with multiple 


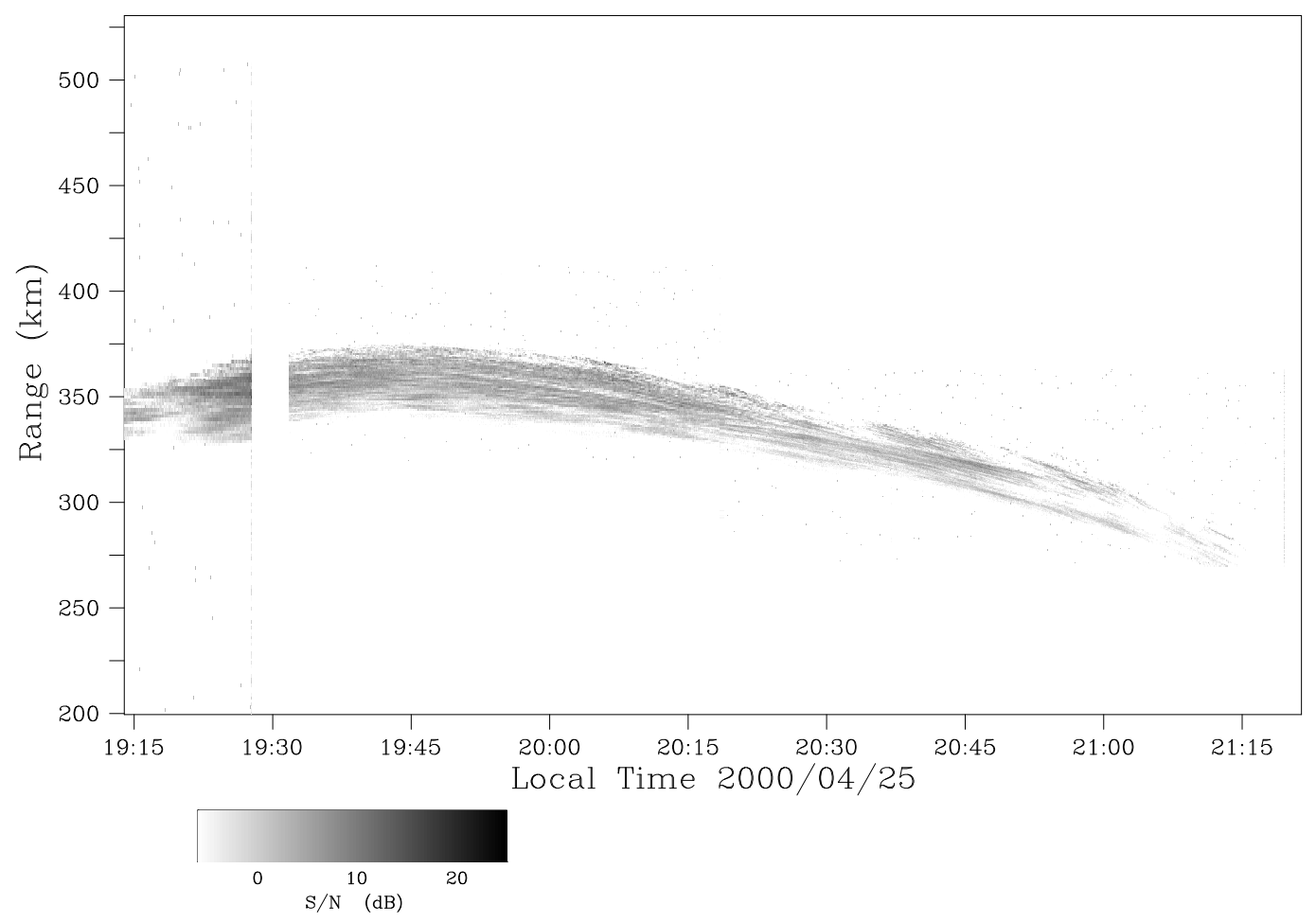

Fig. 1. Range Time Intensity (RTI) representation of a bottom-type scattering layer. Grayscales represent the signal-to-noise ratio on a dB scale. No other F-region irregularities were observed.

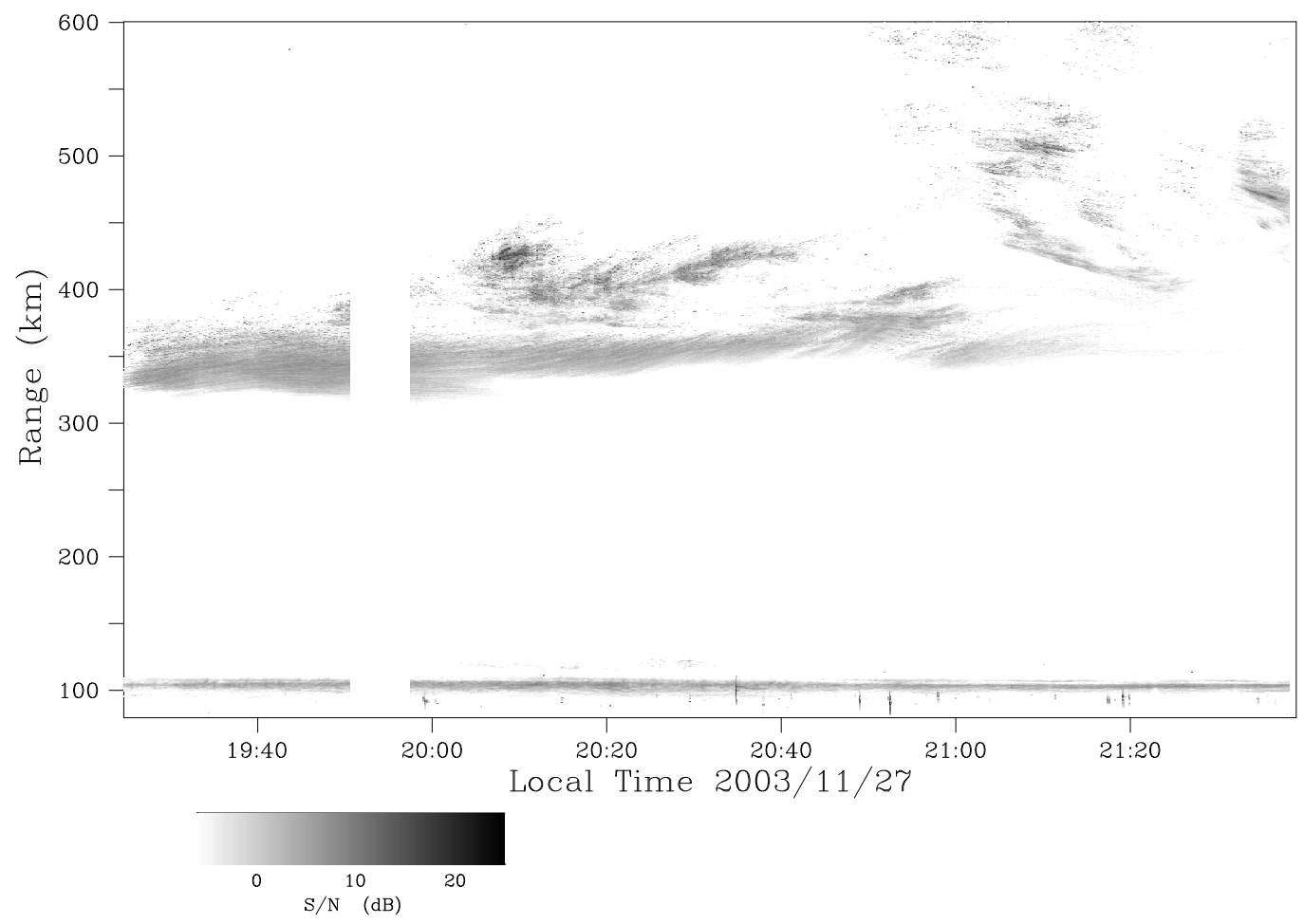

Fig. 2. RTI representation of a bottom-type scattering layer accompanied by emerging radar plumes after 20:00 LT and followed by topside ESF irregularities after 21:00 LT. 

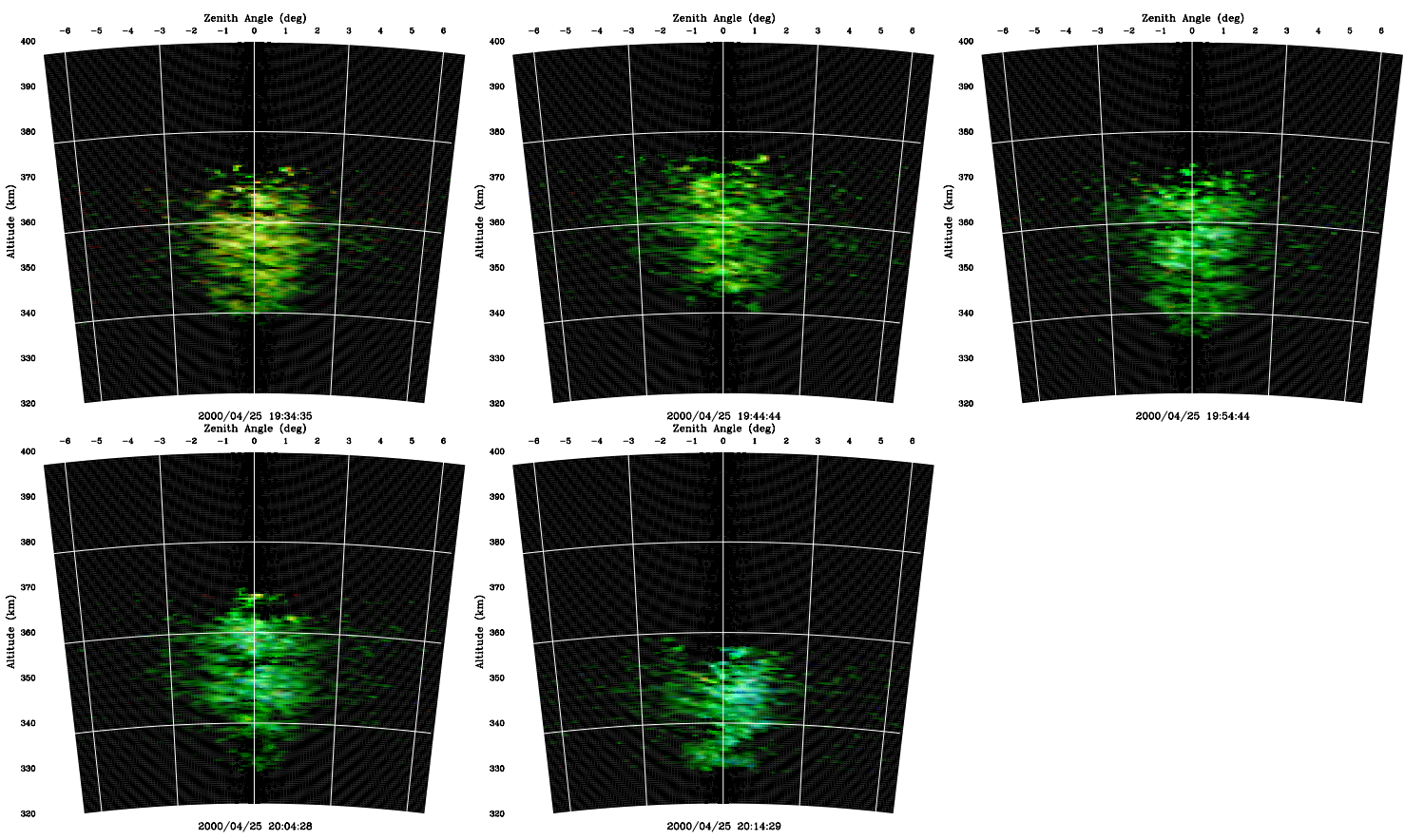

Fig. 3. Aperture synthesis images of the bottom-type layer observed on 25 April 2000 at five local times. Pixel brightness represents the signal-to-noise ratio on a scale from $0-23 \mathrm{~dB}$.

baselines yields multiple moments, and the totality of these moments can be inverted to reconstruct the brightness distribution versus azimuth and zenith angle. The inversion essentially amounts to performing a Fourier transform of the interferometry cross-spectra (Thompson, 1986). However, since the cross-spectra are inevitably sampled incompletely due to the limited number of interferometry baselines available, and because of the presence of statistical fluctuations in the data, the inversion generally must be performed using statistical inverse methods to achieve satisfactory results (Ables, 1974; Jaynes, 1982). For our imaging work, we have employed the MAXent algorithm pioneered for applications in radio astronomy (Wilczek and Drapatz, 1985; Hysell, 1996). This is considered a "super-resolution" method since the resolution of the images it produces is not inherently limited by the Nyquist sampling theorem.

Images of the 25 April 2000 layer are presented in Fig. 3. They were derived from the 15 distinct cross-spectra made available for every range gate by receiving backscatter at Jicamarca using six different spatially separated antennas. The longest available interferometry baseline at Jicamarca is approximately 100 wavelengths. The five images are snapshots computed from 5 -s incoherent integrations and spaced by about $10 \mathrm{~min}$ so as to reflect the behavior of the scattering layer over a $40-\mathrm{min}$ interval. The pixels that make up the images are $450 \mathrm{~m}$ high and $0.1^{\circ}$ wide. The brightness of each pixel reflects the signal-to-noise ratio on a log scale. The hue represents the Doppler shift, with red- and blueshifted echoes being color coded accordingly on a continuous color scale. The saturation represents spectral width, with pure and pastel colors signifying narrow and broad spectra, respectively. We will not derive any conclusions from the spectral moments conveyed by the images here but merely exploit the fact that the colors help highlight some of their morphological features. In general, the spectra of bottomtype layers are usually very narrow, with Doppler shifts that closely match the layer range rate (Woodman and La Hoz, 1976). Note that the transmitting radar antenna beam mainly illuminated regions within $\pm 2^{\circ}$ of zenith for this experiment, explaining why the image peripheries are dark.

The images in Fig. 3 are characterized by narrow, horizontally oriented structures that we interpret as primary plasma waves. The structures are very similar to those seen in radar images of bottomside layers by Hysell (1998), who identified and analyzed them in terms of their dispersion characteristics, except that the latter were vertically oriented. That the structures now in question are horizontally aligned signifies that horizontal wind-driven gradient drift instabilities, as opposed to Rayleigh Taylor or $\boldsymbol{E} \times \boldsymbol{B}$ instabilities, are at work here. (Collisional interchange instabilities are strongly anisotropic in the plane perpendicular to the geomagnetic field and produce waveforms that elongate in the direction normal to the polarization electric field (Zargham and Seyler, 1987). Polarization electric fields are vertically oriented in the case of horizontal wind-driven gradient drift instabilities.) The vertical separation between different primary waves is about $2 \mathrm{~km}$.

Animated sequences of images reveal a vortex-like motion, with irregularities near the top and bottom of the layer moving slowly eastward and rapidly $(\sim 70 \mathrm{~m} / \mathrm{s})$ westward, respectively, and with a clockwise rotation resulting. The primary waves grow in horizontal extent over time. They have 

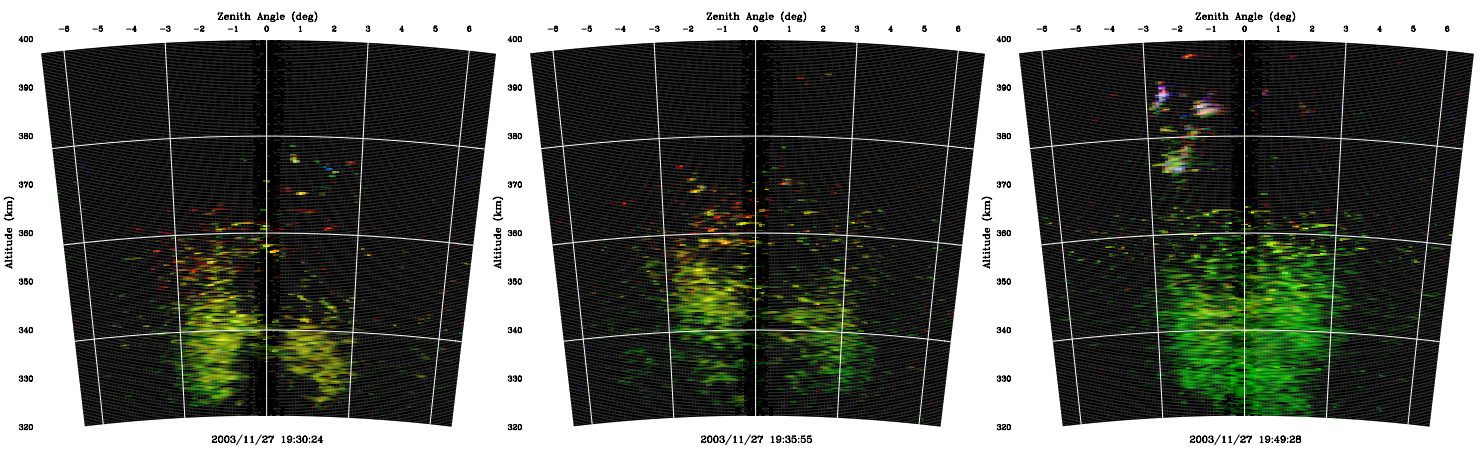

Fig. 4. Aperture synthesis images of the bottom-type layer observed on 27 November 2003 at three local times. Pixel brightness represents the signal-to-noise ratio on a scale from $8-25 \mathrm{~dB}$.

lifetimes longer than their transit time through the radar illuminated volume, and the streaks in the RTI plot in Fig. 1 correspond to their transit across this volume. In this example, no large-scale organization of the irregularities is evident. As primary waves exit one side of the illuminated volume, others enter from the other side essentially continuously.

Radar images of the 27 November 2003 event are shown in Fig. 4. These were derived from data received on eight spatially separated antennas and the 28 associated cross-spectra for every range gate. For this experiment, the transmitting antenna pattern was also broadened so as to effectively illuminate the region within $\pm 4^{\circ}$ of zenith. The resolution of each pixel in the images is $300 \mathrm{~m}$ by $0.1^{\circ}$.

As before, the images show that the bottom-type layers are composed of primary waves oriented nearly horizontally. The vertical separation between adjacent primary waves is only about $1 \mathrm{~km}$ this time. Animated sequences of images indicate that the irregularities all drifted westward at about $100 \mathrm{~m} / \mathrm{s}$ initially but decelerated to a near standstill by 21:00 LT. The point targets in these images occurring above about $350 \mathrm{~km}$ are due to so-called "explosive spread $F$ ", a phenomenon discussed by Woodman and Kudeki (1984) that is unrelated to the present study.

The main difference between the 25 April 2000 and 27 November 2003 data is that the latter show evidence of large-scale horizontal organization. Throughout the entire bottom-type layer event, clusters of intermediate-scale waves, like those shown in Fig. 4, drifted through the radar illuminated volume, separated by a horizontal distance of about $30 \mathrm{~km}$. (The illustrative snapshots in Fig. 4 were chosen so as to depict two different clusters in each frame.) The regions of space with irregularities are about as wide as the regions without, and the $30-\mathrm{km}$ wavelength figure is comparable to the height of the layer thickness. Note that there is no indication of large-scale periodicity in the RTI diagram in Fig. 2 because there is always at least one cluster of irregularities somewhere within the radar illuminated volume.

Starting at about 19:49 LT, small radar plumes began drifting rapidly eastward at altitudes above about $380 \mathrm{~km}$. One of these can be seen in the rightmost image in Fig. 4. These plumes formed to the west of the radar and then drifted eastward overhead. They existed above the shear node and did not appear to be physically connected to the bottom-type irregularities at the time of passage. Their $\sim 100 \mathrm{~m} / \mathrm{s}$ eastward drift rate provides a lower limit for the local thermospheric wind speed.

The irregularities in the bottom-type layer ceased drifting westward by about 20:40 LT and remained nearly stationary within the radar illuminated volume for the next $20 \mathrm{~min}$. This afforded a rare opportunity to observe the long-term, late-stage temporal evolution of the irregularities in detail. Images of the illuminated volume are shown in Fig. 5. In the images, we observe the emergence of large-scale plasma irregularities and radar plumes directly from the bottom-type irregularities. Each of the three emerging radar plumes depicted here appears to have evolved from one of the existing clusters of kilometric primary waves. The spatial separation between the three plumes matches the separation between the pre-existing clusters. The red hue of the most westward of the plumes implies a negative Doppler shift, signifying rapid irregularity ascent. Animated sequences of images, like those in Fig. 5, suggest that a transition from intermediatescale gradient drift waves to large-scale Rayleigh Taylor instabilities was underway.

\section{Discussion}

The main finding of this research is that bottom-type scattering layers in the post-sunset equatorial ionosphere are composed of primary waves excited by horizontal wind-driven gradient drift instabilities rather than the related Rayleigh Taylor or $\boldsymbol{E} \times \boldsymbol{B}$ instabilities. New evidence for this comes from the morphology of the primary waves, which are oriented nearly horizontally rather than vertically. Gradient drift, Rayleigh Taylor, and $\boldsymbol{E} \times \boldsymbol{B}$ instabilities are all variants on the same collisional interchange instability, only with linear growth rates proportional to the forcing functions $u-v$, $g / \nu_{\text {in }}$, and $E / B$, respectively, where $v$ is the plasma drift speed, $u$ is the wind speed, $g$ is gravitation, $v_{\text {in }}$ is the ionneutral collision frequency, $E$ is the background zonal electric field, and $B$ is the magnetic induction. Whereas the 

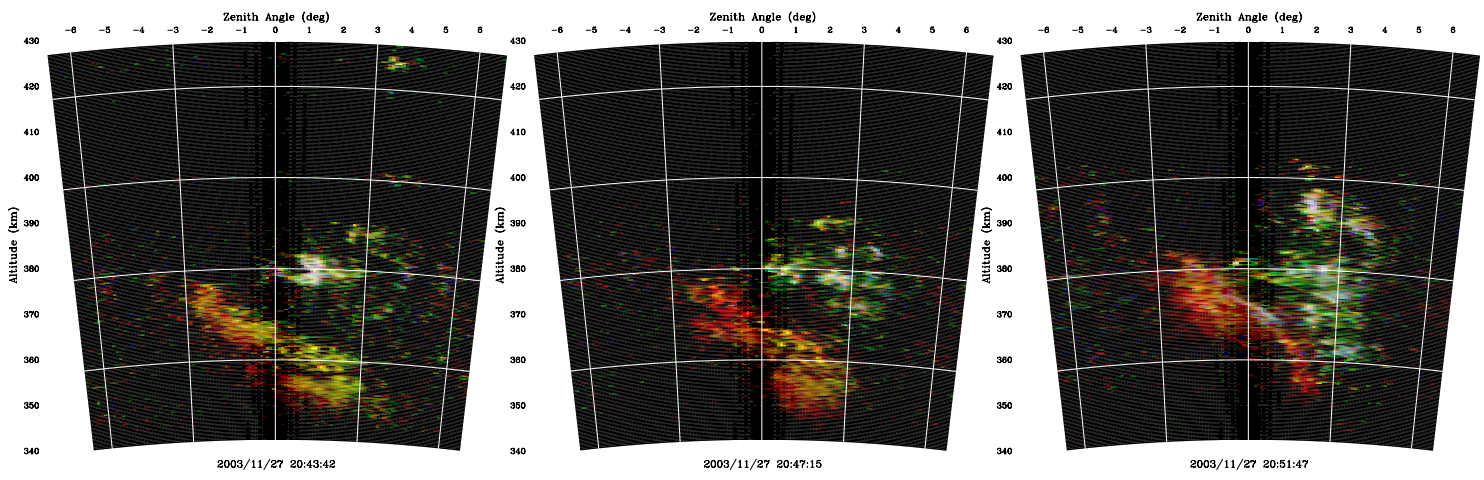

Fig. 5. Images of emerging radar plumes observed on 27 November 2003 at three local times. Pixel brightness represents the signal-to-noise ratio on a scale from $0-23 \mathrm{~dB}$.

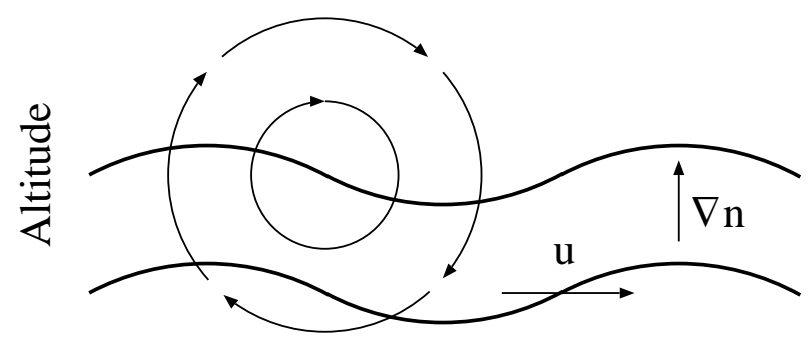

Local time or longitude

Fig. 6. Diagram illustrating the geometry of bottom-type layer generation. The heavy curves are isodensity lines, and the concentric circles represent the circulation associated with the evening vortex. Regions where the horizontal wind is large in the plasma rest frame and antiparallel to a component of the density gradient are gradient drift unstable. Convection due to the evening vortex or another agent produces horizontal conductivity gradients which are alternately stable and unstable.

first of these parameters often assumes a value in excess of $100 \mathrm{~m} / \mathrm{s}$ in the retrograde drifting bottomside F-region strata where the layers reside, the remaining two are generally less than $100 \mathrm{~m} / \mathrm{s}$ and tend to cancel to a significant degree in the bottomside after the evening reversal of the zonal electric field. This was pointed out first by Kudeki and Bhattacharyya (1999) in their interpretation of the layers.

In the 25 April 2000 event, primary gradient drift waves were observed continuously, undergoing vortex motion in a bottomside shear layer. These observations support the picture described by Kudeki and Bhattacharyya (1999), in which the evening vortex creates both the differential ionneutral horizontal drift and the horizontal conductivity gradients necessary to support gradient drift instabilities. No large-scale organization of the irregularities was evident, and no topside irregularities were observed.

However, the 27 November 2003 observations showed that the gradient drift instabilities can be modulated, occurring in clusters and exhibiting large-scale horizontal periodicity. We surmise that a large-scale horizontal wave in the plasma density existed in the bottomside F-region during this event. The horizontal conductivity gradients associated with the wave would be alternately stable and unstable to wind-driven gradient drift instability, effectively turning the process on and off in alternating phases of the wave (see Fig. 6). Periodicity is not evident in radar RTI data from Jicamarca and requires imaging techniques to be discerned.

The fact that retrograde drifts can persist in the bottomside F-region for several hours after the evening reversal of the zonal electric field suggests that the E-region dynamo (and not just electrojet currents closing near the terminator) can drive them. A nonlocal analysis in the Appendix of this manuscript shows how gradient drift waves with transverse wavelengths of a few kilometers or less can decouple from and thereby avoid being shorted out by the E-region conductivity required by the dynamo. Large-scale Rayleigh Taylor instabilities will experience damping under the influence of significant E-region conductivity, however, implying that the two classes of waves cannot coexist on the same flux tube for very long. Large-scale waves can grow on flux tubes with apex heights above the bottom-type layers, however, and seem to have done so throughout the 27 November 2003 event.

Late in that event, large-scale instabilities and plume structures began to emerge directly from the bottom-type layer as the retrograde drift was ceasing and just prior to the layer's disappearance. The plumes shared the horizontal scale size of the initial irregularity clustering. We further surmise that the large-scale waves present throughout the event served as a seed irregularity for large-scale generalized Rayleigh Taylor instability. Large-scale instability was unfavorable earlier in the event when the E-region dynamo dominated bottomside F-region electrodynamics and when intense kilometric waves were a source of anomalous diffusion. The emergence of large-scale instabilities very often occurs at the same time the retrograde drifts stop and the bottom-type layers dissipate, just as in this case (Hysell and Burcham, 1998).

The picture outlined here suggests that it may be possible to monitor the distribution of irregularities within 
bottom-type layers as a diagnostic of large-scale perturbations in the bottomside F-region ionosphere that could serve as seed irregularities for ESF. The predictive utility of this strategy is supported by a few other data sets exhibiting the phenomenology described here and will be evaluated more comprehensively in future experiments. The $30-\mathrm{km}$ wavelength of the large-scale waves inferred from this analysis is at the short wavelength extreme for gravity waves thought to be capable of propagating into the thermosphere, and it is possible that the bottom-type layers may be a telltale sign of gravity wave seeding. However, the fact that this wavelength is also comparable to the depth of the shear layer suggests a causal relationship between the large-scale waves and the shear itself. In a follow-on paper, Hysell and Kudeki (2004) argue that a collisional shear instability in the bottomside F-region can produce large-scale seed waves in the layers. In that case, monitoring the shear may ultimately provide a practical means of forecasting spread $F$.

\section{Appendix A}

Here, we develop and solve an eigenvalue problem describing collisional interchange mode plasma waves with plane wave characteristics perpendicular to the geomagnetic field and with variations along the magnetic field. The derivation is related to the calculation performed by Farley (1959). We can show that F-region waves with sufficiently large perpendicular wave numbers can be electrically decoupled from the $\mathrm{E}$ and valley regions and therefore remain undamped by conductivity present at the feet of the magnetic field lines. This calculation extends upon the one presented by Hysell (2000) by including magnetic field curvature effects.

We adopt an orthogonal $(x, p, q)$ coordinate system, where $x$ is eastward, $p$ is parallel to the geomagnetic field, and $q$ is normal to $x$ and $p$. We then write the current density $\mathbf{J}$ in the plasma as

$\mathbf{J}=n e \mu_{\perp} \mathbf{E}_{x}+n e \mu_{\|} \mathbf{E}_{p}$,

where $n$ is the plasma number density, $e$ is the electron charge magnitude, $\mathbf{E}$ is the electric field, and $\mu_{\perp}$ and $\mu_{\|}$are the perpendicular and parallel plasma mobilities, respectively. Hall mobility is neglected in this analysis. The current density is linearized according to the following prescriptions:

$\mathbf{E}(x, p, q)=E_{\circ} \hat{x}-\nabla \phi(p) e^{i(\omega t-k x)}$

$n(x, p, q)=n_{\circ}(p, q)+n_{1}(p) e^{i(\omega t-k x)}$

where $n_{\circ}(p, q)$ and $E_{\circ}$ are equilibrium values, and $\phi$ and $n_{1}$ are the perturbed electrostatic potential and number density, varying with $x$ and $p$ as indicated. Note that the background parameters do not vary with $x$. Note also that, while this parameterization is strictly appropriate for waves produced by Rayleigh Taylor instabilities, we could equally well analyze horizontal wind-driven gradient drift instabilities by exchanging the roles of $x$ and $q$ without affecting the conclusions of the analysis.
The condition that the current density be solenoidal in the plasma leads to the following relationship between the $n_{1}$ and $\phi$ (see Hysell, 2000, for details):

$n_{1}=\frac{1}{i k E_{\circ}}\left[n_{\circ} k^{2} \phi-\frac{1}{\mu_{\perp}} \frac{1}{h_{p} h_{q}} \frac{\partial}{\partial p}\left(\frac{h_{q}}{h_{p}} n_{\circ} \mu_{\|} \frac{\partial \phi}{\partial p}\right)\right]$,

in which $h_{p}(p, q)$ and $h_{q}(p, q)$ are the scale factors associated with the coordinate system and are discussed in more detail below. For orthogonal coordinate systems, the scale factor is the differential physical length swept out by a differential change in the associated coordinate.

A second equation to close the system is provided by ion continuity, which may be written as:

$\gamma n_{1}+\frac{i k n_{\circ}}{B l} \phi=0$,

where $\gamma$ is the growth rate (imaginary part of frequency) and $B$ is the magnetic induction, taken to be constant. In writing this equation, we take the dominant perturbation vertical velocity to be due to $\boldsymbol{E} \times \boldsymbol{B}$ drifts driven by the wave and also suppose that the $q$ (vertical) derivative of the background number density can be expressed in terms of the single scale height parameter $l$. Combining this result with the result of the quasi-neutrality condition yields the desired eigenvalue problem.

$\frac{\partial^{2} \phi}{\partial p^{2}}+\frac{1}{g} \frac{\partial g}{\partial p} \frac{\partial \phi}{\partial p}+k_{\circ}^{2} h_{p}^{2} \frac{\mu_{\perp}}{\mu_{\|}} \phi=0$,

in which the following auxiliary variables appear

$$
\begin{aligned}
g & \equiv\left(h_{q} / h_{p}\right) n_{\circ} \mu_{\|} \\
k_{\circ}^{2} & \equiv k^{2}\left(\frac{\gamma_{\circ}}{\gamma}-1\right)
\end{aligned}
$$

and where $\gamma_{\circ} \equiv E_{\circ} / l B$ is the well-known linear, local growth rate for collisional interchange instabilities driven by a background electric field. (This term could equally well include gravity and wind drivers for the generalized Rayleigh Taylor instability.)

The significance of the eigenvalue $k_{\circ}^{2}$ is made clear by solving Eq. (2) for the growth rate as follows:

$\gamma=\frac{\gamma_{\circ}}{1+k_{\circ}^{2} / k^{2}}$.

Evidently, the growth rate of a wave can only be comparable to its local growth rate estimate if its transverse wave number $k$ is comparable to or greater than $k_{\circ}$, which therefore serves as an effective cutoff wave number for a given mode. The situation is analogous to waveguide cutoff.

The boundary conditions on Eq. (A1) are chosen to reflect an instability driven mainly at the magnetic field apex point and damped at the feet of the field lines. We therefore take the potential to have a maximum at the apex point (here $350 \mathrm{~km}$ altitude) and a zero at a nominal altitude of $125 \mathrm{~km}$. The eigenvalues do not turn out to be very sensitive to the altitudes chosen. 


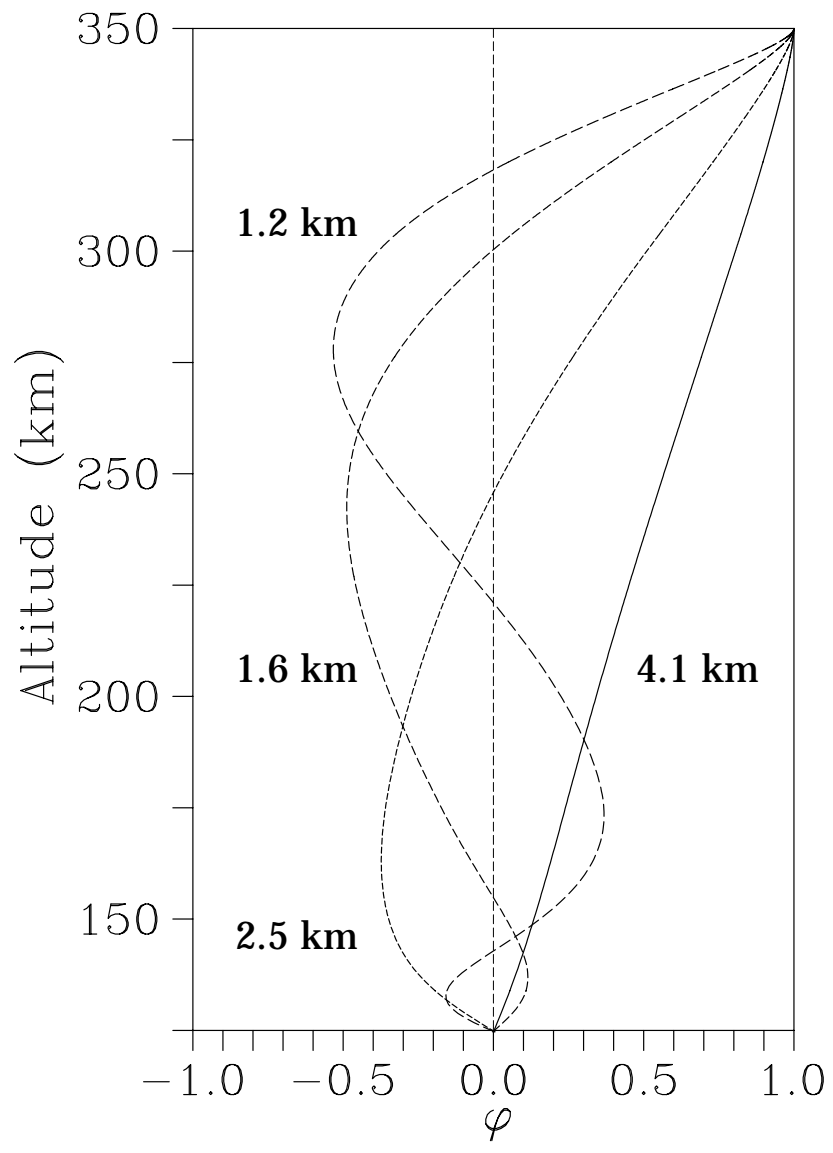

Fig. A1. Mode shapes for the four lowest order eigenmodes. The horizontal axis represents relative electrostatic potential, and the vertical axis represents altitude on a magnetic field line with an apex height of $350 \mathrm{~km}$. The relative potential is greatest at the apex point. Labels represent the cutoff wavelengths, $2 \pi / k_{\circ}$, in $\mathrm{km}$.

The coordinate system is a magnetic dipole system. A conventional choice for such coordinates is defined by $r / R_{e}=p \sin ^{2} \theta=\sqrt{(\cos \theta)} / q^{\prime}$, where $r$ and $\theta$ are radial distance and colatitude, respectively, $R_{e}$ is the Earth's radius, and $p$ is the McIlwain parameter. This coordinate system is well behaved at the equator in the sense that both $p$ and $q^{\prime}$ are finite there. However, the scale factor $h_{q^{\prime}}$ can be shown to be zero at the magnetic equator, posing problems for the numerical solution of differential equations there. Consequently, we instead utilize the coordinate $q \equiv q^{\prime 2}$ in our calculations, where $q$ is the magnetic scalar potential. The associated scale factors can then be shown to be:

$h_{p}^{2}=R_{e}^{2} \frac{\sin ^{6} \theta}{1+3 \cos ^{2} \theta}$

$h_{q}^{2}=R_{e}^{2} \frac{\left(r / R_{e}\right)^{6}}{1+3 \cos ^{2} \theta}$

such that the differential lengths $d s^{2}=h_{p}^{2} d p^{2}+h_{q}^{2} d q^{2}$. Not only are the scale factors finite at the magnetic equator, they also vary slowly with colatitude and altitude there, meaning that the grid squares used for computation of multidimen- sional differential equations can be nearly constant, facilitating efficient use of computational resources.

The mode shapes for the four lowest order eigenmodes of Eq. (A1) were computed using a second-order Runge Kutta method and plotted in Fig. A1. These were calculated using parameters derived from the IRI2001 and MSIS90E models for the longitude of Jicamarca and for conditions representative of December solstice, 20:00 LT, during moderate solar flux conditions.

The labels on Fig. A1 are the corresponding eigenvalues, written as cutoff wavelengths, i.e. $2 \pi / k_{\circ}$. These are the longest transverse wavelengths that the unstable waves can have without suffering significant damping due to conductivity in the $\mathrm{E}$ and valley regions. The values shown are of the order of a few kilometers.

Acknowledgements. D. L. Hysell wishes to thank E. Kudeki for valuable discussions. This work was supported by the National Science Foundation through cooperative agreement ATM-9911209 to Cornell University and by NSF grant ATM-0225686 and NASA grant NAG5-12164 to Cornell University. The Jicamarca Radio Observatory is operated by the Instituto Geofísico del Perú, Ministry of Education, with support from the NSF cooperative agreements just mentioned. The help of the staff was much appreciated.

Topical Editor M. Lester thanks C. Haldoupos and another referee for their help in evaluating this paper.

\section{References}

Ables, J. G.: Maximum entropy spectral analysis, Astron. Astrophys. Suppl. Ser., 15, 383, 1974.

Farley, D. T.: A theory of electrostatic fields in a horizontally stratified ionosphere subject to a vertical magnetic field, J. Geophys. Res., 64, 1225, 1959.

Farley, D. T., Ierkic, H. M., and Fejer, B. G.: Radar interferometry: A new technique for studying plasma turbulence in the ionosphere, J. Geophys. Res., 86, 1467, 1981.

Flaherty, J. P., Seyler, C. E., and Trefethen, L. N.: Large-amplitude transient growth in the linear evolution of equatorial spread $\mathrm{F}$ with a sheared zonal flow, J. Geophys. Res., 104, 6843, 1999.

Fu, Z. F., Lee, L. C., and Huba, J. D.: A quasi-local theory of the $\boldsymbol{E} \times \boldsymbol{B}$ instability in the ionosphere, J. Geophys. Res., 91, 3263, 1986.

Guzdar, P. N., Satyanarayana, P., Huba, J. D., and Ossakow, S. L.: Influence of velocity shear on Rayleigh-Taylor instability, Geophys. Res. Lett., 9, 547, 1983.

Haerendel, G., Eccles, J. V., and Cakir, S.: Theory for modeling the equatorial evening ionosphere and the origin of the shear in the horizontal plasma flow, J. Geophys. Res., 97, 1209, 1992.

Hysell, D. L.: Radar imaging of equatorial $F$ region irregularities with maximum entropy interferometry, Radio Sci., 31, 1567, 1996.

Hysell, D. L.: Imaging coherent scatter radar studies of bottomside equatorial spread F, J. Atmos. Sol. Terr. Phys., 60, 1109, 1998.

Hysell, D. L.: A review and synthesis of plasma irregularities in equatorial spread F, J. Atmos. Sol. Terr. Phys., 62, 1037, 2000.

Hysell, D. L. and Burcham, J.: JULIA radar studies of equatorial spread F, J. Geophys. Res., 103, 29 155, 1998.

Hysell, D. L. and Kudeki, E.: Collisional shear instability in the eqautorial f region ionosphere, J. Geophys. Res., in press, 2004. 
Hysell, D. L. and Seyler, C. E.: A renormalization group approach to estimation of anomalous diffusion in the unstable equatorial $\mathrm{F}$ region, J. Geophys. Res., 103, 26731, 1998.

Jaynes, E. T.: On the rationale of maximum-entropy methods, Proc. IEEE, 70, 939, 1982.

Kudeki, E. and Bhattacharyya, S.: Post-sunset vortex in equatorial F-region plasma drifts and implications for bottomside spread-F, J. Geophys. Res., 28, 28 163, 1999.

Kudeki, E. and Sürücü, F.: Radar interferometric imaging of fieldaligned plasma irregularities in the equatorial electrojet, Geophys. Res. Lett., 18, 41, 1991.

Kudeki, E., Farley, D. T., and Fejer, B. G.: Long wavelength irregularities in the equatorial electrojet, Geophys. Res. Lett., 9, 684, 1982.

Perkins, F. W. and Doles III, J. H.: Velocity shear and the ExB instability, J. Geophys. Res., 80, 211, 1975.

Satyanarayana, P., Guzdar, P. N., Huba, J. D., and Ossakow, S. L.: Rayleigh-Taylor instability in the presence of a stratified shear layer, J. Geophys. Res., 89, 2945, 1984.
Thompson, A. R.: Interferometry and Synthesis in Radio Astronomy, John Wiley, New York, 1986.

Tsunoda, R. T.: Time evolution and dynamics of of equatorial backscatter plumes, 1, Growth phase, J. Geophys. Res., 86, 139, 1981.

Wilczek, R. and Drapatz, S.: A high accuracy algorithm for maximum entropy image restoration in the case of small data sets, Astron. Astrophys., 142, 9, 1985.

Woodman, R. F. and Kudeki, E.: A causal relationship between lightning and explosive spread F, Geophys. Res. Lett., 11, 1165, 1984.

Woodman, R. F. and La Hoz, C.: Radar observations of F region equatorial irregularities, J. Geophys. Res., 81, 5447, 1976.

Zargham, S.: Numerical simulations of the ionospheric interchange instability, Ph.D. thesis, Cornell University, Ithaca, N.Y., 1988.

Zargham, S., and Seyler, C. E.: Collisional interchange instability, 1, Numerical simulations of intermediate-scale irregularities, J. Geophys. Res., 92, 10 073, 1987.

Zargham, S. and Seyler, C. E.: Collisional and inertial dynamics of the ionospheric interchange instability, J. Geophys. Res., 94, 9009, 1989. 